\title{
Evaluation of Binarization Methods Using Global Threshold for Chinese Rubbing Images
}

\author{
Zhi-Kai Huang ${ }^{1}$, Jun-Mei Xi ${ }^{1}$ \\ 1.College of Mechanical \& Electrical Engineering, \\ Nanchang Institute of Technology \\ Nanchang, Jiangxi 330099, China \\ huangzhik2001@163.com
}

\author{
Fang Wang ${ }^{1}$, Han Huang ${ }^{2}$ \\ 2.School of Mechanical and Engineering \\ Harbin Institute of Technology \\ Harbin, Heilongjiang, 150080, China \\ 863503892@qq.com
}

\begin{abstract}
There are a huge amount of historical Chinese rubbing images in different library that have not been exploited electronically. However, many invaluable collections of rubbing images are already digitized and indexed for consulting, exchange and distant access purposes which protect them from direct manipulation. In many applications of digital rubbing image, the use of binary images can decrease the computational cost of the succeeding steps compared to using gray-level images. Thresholding is a simple but effective tool to separate characters from the background for rubbing image. In this paper, five global thresholding algorithms such as Histogram Isodata method, Minimum Cross Entropy(MCE) method, Kittler's method, Maxium Entropy method,Ostu's method and Fuzzy CMeans(FCM) clustering thresholding method that had been used widely by scholars have been researched. Two quantitative measure of comparison is provided by the Peak Signal to Noise Ratio (PSNR) and Mean Square Error (MSE) of the rubbing image for segmentation results.
\end{abstract}

Keywords-Thresholding;Chinese rubbing image, Ostu's thresholding; image segmentation; FCM

\section{INTRODUCTION}

With the computer technology and the rapid development of communication technology, modern society has gradually entered into the information age. The decreasing cost of digital image capture and processing hardware, especially CCD-scanned (Charge Coupled Device) photodiode arrays and memory chips, has made it possible to consider approaches to OCR (Optical Character Recognition) that have not been practical before. The traditional record information, storing the information carrier (such as papered) has been unable to meet those rapid require [1]. Much valuable information, such as Chinese calligraphy, art works and others, is usually scanned into digital document images to be stored, transmitted, displayed and printed. In order to ensure the effectively process of the document image, document image segmentation research is particularly important. At the same time, document image binarization is an essential preprocessing step in many document-related applications, such as cleanup, storage, transmission, and offline analysis and recognition. In many applications of image processing, the use of binary images can decrease the computational cost of the succeeding steps compared to using gray-level images. Thresholding is a simple but effective tool to separate characters from the background for rubbing image. Depending on the application, the foreground can be represented by gray-level 0 , that is, black as for text, and the background by the highest luminance for document paper that is 255 in 8-bit images or conversely the foreground by white and the background by black[2][3]. At the same time, the global thresholding approach is less sensitive to noise and does not require elaborate enhancement which is usually sensitive to the individual image characteristics and requires a lot of supervision. But, there is that the lack of objective measures to assess the performance of various thresholding algorithms for rubbing image segmentation. The main difficulty of image thresholding could be come from various factors, such as non-stationary and correlated noise, ambient illumination, busyness of gray levels within the object and its background, inadequate contrast, and object size not commensurate with the scene, complicate the thresholding operation [4].

In this study, we compared five global thresholding algorithms, such as histogram shape information thresholding (Iterative Isodata method) [5], clustering of gray-level information thresholding (Otsu's image thresholding [6], Kittler and Illingworth's minimum cross entropy thresholding), entropy-based thresholding methods (Maximum entropy thresholding)[7] and Fuzzy C-Means clustering thresholding method[8]. A quantitative measure of comparison is provided by the PSNR (Peak Signal to Noise Ratio) of the image. The remainder of the paper is broken down into 4 sections. In Sections 2, we reviewed the five thresholding method. Some results of experiments have been shown in section 3.The conclusion and future works have been introduced in section 4 .

\section{THE THEORY OF RELATED METHODS}

In here, we may consider Chinese rubbing image segmentation as a specific binarization procedure which tries to keep only text and other objects which in this paper. Improper thresholding causes blotches, streaks, and erasures on the document, confounding segmentation and recognition tasks. Since digital image segmentation using thresholding technique is a well-researched field, there exist many algorithms for determining an optimal threshold of the image. The detail of those methods could be found in [10].

\section{A. Histogram shape information thresholding}

The basic and easiest way to determine the global threshold of image in the case of binary image is based on finding two peaks of normal distribution from image 
histogram separated by a valley corresponding to the intermediate gray levels, which is used to divide the object and background into bi-level. Pixels with a gray scale level under the threshold level are labeled as print; pixels with a gray scale level above the threshold level are labeled as background. The process can be described as follows:

$$
I(x, y)=\left\{\begin{array}{lll}
1 & \text { if } & f(x, y) \leq T \\
0 & \text { otherwise }
\end{array}\right.
$$

Concrete steps of this algorithm could be described as follow:

1) Select an initial estimate for $T$.

2) Segment the image using $T$. This will produce two groups of pixels. $G 1$ consisting of all pixels with gray level values $>T$ and $G 2$ consisting of pixels with values $<=T$.

3) Compute the average gray level values $\mu_{1}$ and $\mu_{2}$ for the pixels in regions $G 1$ and $G 2$.

4) Compute a new threshold value, $T=\frac{1}{2}\left(\mu_{1}+\mu_{2}\right)$.

5) Repeat steps 2 through 4 until difference in $T$ in successive iterations is smaller than a predefined parameter $T_{0}$.

\section{B. OTSU's thresholding method}

Otsu has developed a thresholding method based on a discriminate criterion, which is the ratio of between-class variance and total variance of gray levels. Let the pixels of a given image. Let the pixels of a given image be represented in $L$ gray levels $\left[1,2^{L}\right]$. The number of pixels at level $i$ is denoted by $n_{i}$, and the total number of pixels by

$$
N=n_{1}+n_{2}+\cdots+n_{i}
$$

So, a probability of gray level $i$ in an image is

$p_{i}=n_{i} / N$. Then suppose that the pixels were dichotomized into two classes $C_{1}$ and $C_{2}$, which denote pixels with levels $[1, k]$ and $[k+1, L]$, respectively.

Then, the gray level probability distributions for the two classes are

$$
\begin{array}{rc}
C_{1}: & p_{1} / \omega_{1}(t), \cdots, p_{t} / \omega_{1}(t) \\
C_{2}: & p_{t+1} / \omega_{2}(t), \cdots, p_{L} / \omega_{2}(t) \\
\text { where } \omega_{1}(t)=\sum_{i=1}^{k} P_{i} \text { and } \omega_{2}(t)=\sum_{i=k+1}^{L} P_{i} .
\end{array}
$$

Also, the means for classes $C_{1}$ and $C_{2}$ are

$$
\mu_{1}=\sum_{i=1}^{k} i p_{i} / \omega_{1}(t) \text { and } \mu_{2}=\sum_{i=1}^{k} i p_{i} / \omega_{2}(t) \text {. }
$$

Let $\mu_{T}$ be the mean intensity for the whole image. It is easy to show that

$$
\begin{gathered}
\omega_{1} \mu_{1}+\omega_{2} \mu_{2}=\mu_{T} \\
\omega_{1}+\omega_{2}=1
\end{gathered}
$$

Based on discriminate analysis, Ostu's method of threshold selection is ranked as the best and fastest global thresholding method [16], [17]. This method uses a combination of a variety of segmentation methods, while ensuring the speed greatly improved the accuracy of image segmentation; the resulting document image binarization results are very satisfactory.

\section{Minimum error thresholding}

Minimum error thresholding method finds the optimum threshold by optimizing the average pixel classification error rate directly, using either exhaustive search or an iterative algorithm. This method assumes that an image is characterized by a mixture distribution with the population of object and background classes are normally distributed. The probability density function of the mixture distribution is estimated based on the histogram of the image.

In this method assumes that the image can be characterized by a mixture distribution of foreground and background

pixels: $\quad p(g)=P(T) \cdot p_{f}(g)+[1-P(T)] \cdot p_{b}(g)$

considers equal variance Gaussian density functions, and minimizes the total misclassification error via an iterative search. The total misclassification error expression can be interpreted also as a fitting error expression to be minimized such that:

$$
\begin{aligned}
& T_{o p t}=\arg \min \left[P(T) \log \sigma_{f}(T)+\right. \\
& (1-P(T)) \log \sigma_{b}(T)-P(T) \log P(T) . \\
& -(1-P(T)) \log (1-P(T))]
\end{aligned}
$$

where $\sigma_{f}(T)$ and $\sigma_{b}(T)$ are, respectively, the foreground and background variances for each choice of $T$.

Besides assuming a normal distribution, the approach also assumes that the overlap between the two underlying distributions is small and the truncation error in the derivation of the algorithm can thus be ignored.

\section{Fuzzy C means thresholding}

Among all image segmentation methods, clustering methods get more attention in image segmentation field. Clustering is considered as the process of classifying patterns such that the patterns in the same cluster are more similar than ones in other clusters. In the clustering method, fuzzy C-means (FCM) algorithm is one of the most widely used methods due to its flexibility advantage in classifying pixels of segmented image [14]. The FCM algorithm assigns pixels to each category by using fuzzy memberships.

Let $X=\left(x_{1}, x_{2}, \cdots, x_{N}\right)$ denotes an image with $\mathrm{N}$ pixels to be partitioned into c clusters, where $x_{i}$ represents features data [11]. However, the spatial Fuzzy c-means (FCM) is a method of clustering which allows one piece of data to belong to two or more clusters. This method (developed by Dunn in 1973 and improved by Bezdek in 1981) is frequently used in pattern recognition [8] [9]. The algorithm is an iterative optimization that minimizes the of the objective function $J_{q}(U, V)$ as follows:

$$
J_{q}(U, V)=\sum_{k=1}^{n} \sum_{i=1}^{c}\left(u_{i k}\right)^{q} d^{2}\left(x_{k}, v_{i}\right)
$$


where $X=\left\{x_{1}, x_{2}, \ldots, x_{n}\right\} \subseteq R^{p}, n$ is the number of data items, $c$ is the number of clusters with $2 \leq c<n$, $u_{i k}$ is the degree of membership of $x_{k}$ in the $i^{t h}$ cluster, $q$ is a weighting exponent on each fuzzy membership, $v_{i}$ is the prototype of the centre of cluster $i, d^{2}\left(x_{k}, v_{i}\right)$ is a distance measure between $x_{k}$ object and cluster centre $v_{i}$. A solution of the object function can be obtained via an iterative process, which is carried as follows:

1) Set value for $c, q$ and $\varepsilon$.

2) Initialize the fuzzy partition matrix $U$.

3) Set the loop counter $b=0$.

4) Calculate the $c$ cluster centers $\left\{v_{i}^{(b)}\right\}$ with $U^{(b)}$ :

$$
v_{i}^{(b)}=\frac{\sum_{k=1}^{n}\left(u_{i k}^{(b)}\right)^{q} x_{k}}{\sum_{k=1}^{n}\left(u_{i k}^{(b)}\right)^{q}}
$$

5) Calculate the membership $U^{(b+1)}$. For $k=1$ to $n$, calculate the following:

$$
\begin{aligned}
& I_{k}=\left\{i \mid 1 \leq i \leq c, d_{i k}=\left\|x_{k}-v_{i}\right\|=0\right\} \\
& \tilde{I}_{k}=\{1,2, \ldots, c\}-I_{k}
\end{aligned}
$$

for the $k^{\text {th }}$ column of the matrix, compute new membership values:

a) if $I_{k}=\phi$, then $u_{i k}^{(b-1)}=\frac{1}{\sum_{j=1}^{c}\left(\frac{d_{i k}}{d_{j k}}\right)^{2 /(q-1)}}$,

else $u_{i k}^{(b-1)}=0$ for all $i \in I_{k}$ and $\sum_{i \in I_{k}} u_{i k}^{(b-1)}=1$;

next $k$.

6) If $\left\|U^{(b)}-U^{(b-1)}\right\|<\varepsilon$, stop; otherwise, set $b=b+1$ and go to step 4 .

\section{E. Max entropy method}

In this method the foreground and background classes are considered as two different sources. When the sum of the two class entropies is a maximum the image is said to be optimally thresholded. Thus using the definitions of the foreground and background entropies,

$$
\begin{aligned}
H_{f}(T)= & -\sum_{g=0}^{T} \frac{p(g)}{P(T)} \log \frac{p(g)}{P(T)} \\
H_{b}(T)= & -\sum_{g=T+1}^{G} \frac{p(g)}{P(T)} \log \frac{p(g)}{P(T)} \text { one has: } \\
& T_{\text {opt }}=\arg \max \left[H_{f}(T)+H_{b}(T)\right]
\end{aligned}
$$

\section{EXPERIMENTAL RESULTS PERFORMANCE EVALUATION}

To investigate the performance of the different threshold method, the images which come from library of Harvard are chosen as input images with resolution of $2112 * 1781$ (another rubbing image size is $1688 * 2400$ ) pixels and 256 intensity levels, respectively. For the purpose of comparison, we adopt the same approach that was taken by Kittler and Illingworth, Ostu's method, the minimum error method, and fuzzy C-means thresholding method are implemented on the Chinese rubbing image as stored in Harvard library, could be found in reference [12].

The rubbing image histograms are shown in Fig .2 and 4. The exact thresholds that have been obtained by the five algorithms are summarized in Table 1, repectively. The results of Peak Signal to Noise Ratio (PSNR) and Mean Square Error (MSE) for different algorithm have been shown in Table 1 also.

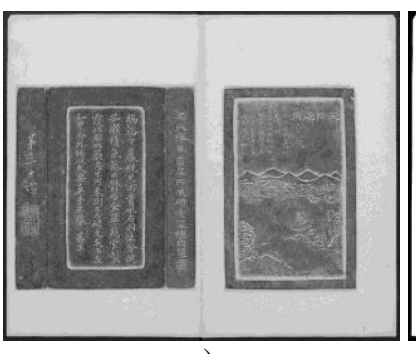

a)

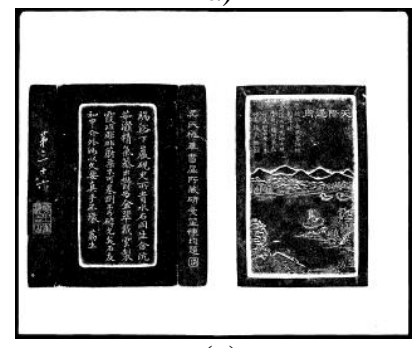

(c)

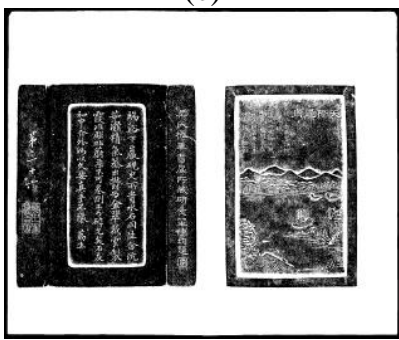

(e)

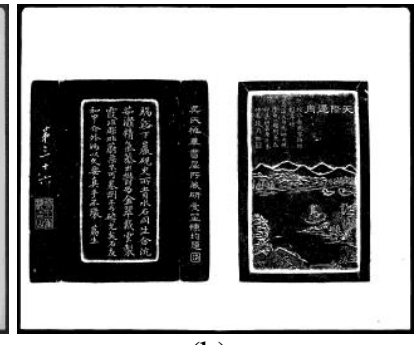

(b)

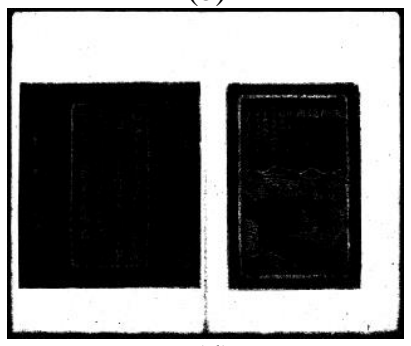

(d)

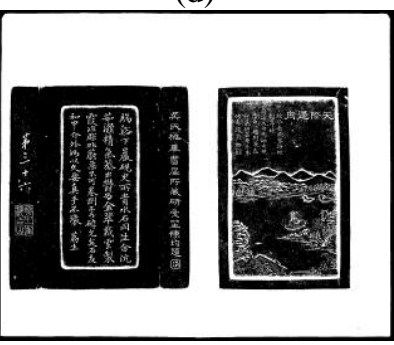

(f)

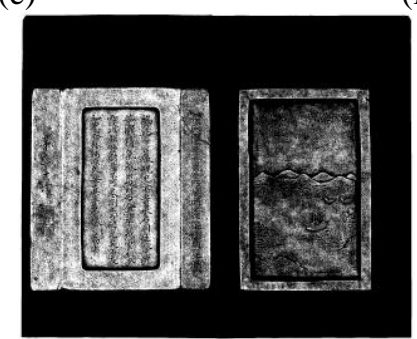

(g)

Figure 1. Thresholding results of rubbing image which number is 10402096 [12]. From left to right and from top to bottom: Original images, segmentation image using isodata thresholding, segmentation image using Minimum cross entropy thresholding image, segmentation image using Kittler's method thresholding, segmentation image using Max entropy method thresholding, segmentation image using Ostu's method thresholding, segmentation image using FCM's method thresholding. 


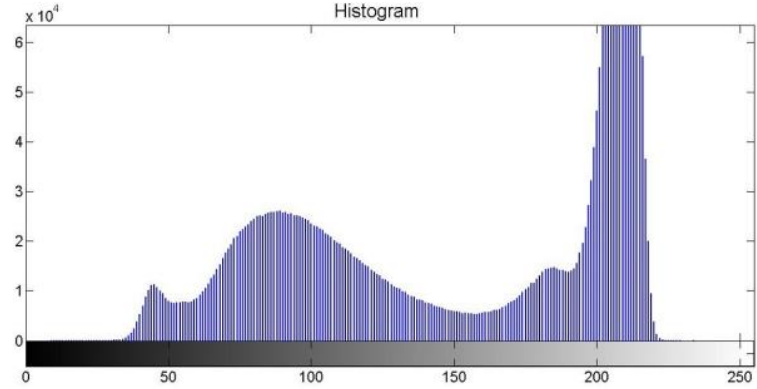

Figure 2. Histogram of rubbing image that No. 12443747

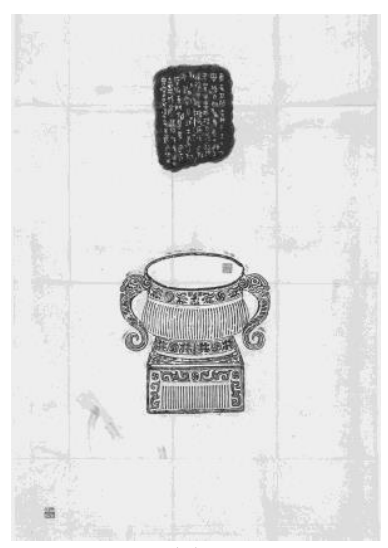

(a)

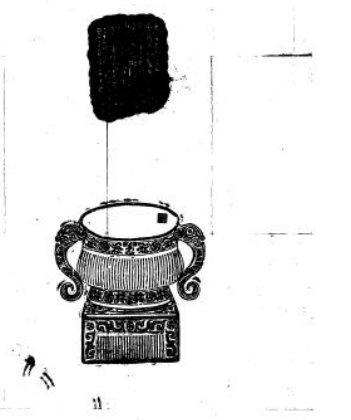

(c)

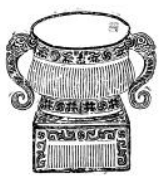

(d)
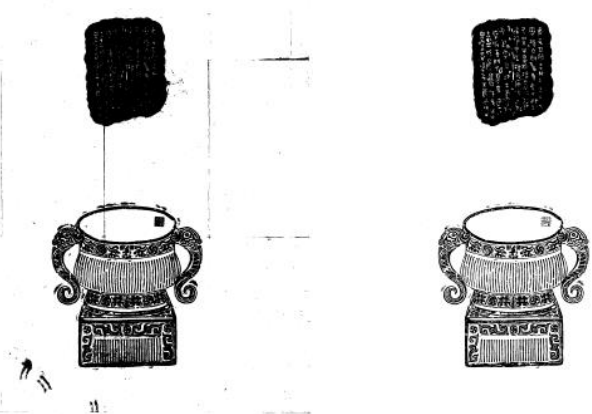

(e)

(f)

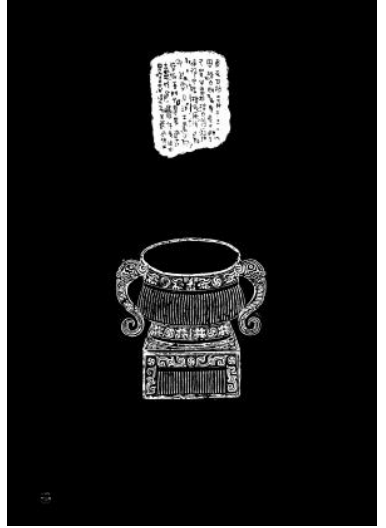

(g)

Figure 3. Thresholding results of rubbing image which number is 14940418 [13]. From left to right and from top to bottom: Original images, segmentation image using isodata thresholding, segmentation image using Minimum cross entropy thresholding image, segmentation image using Kittler's method thresholding, segmentation image using Max entropy method thresholding, segmentation image using Ostu's method thresholding, segmentation image using FCM's method thresholding.

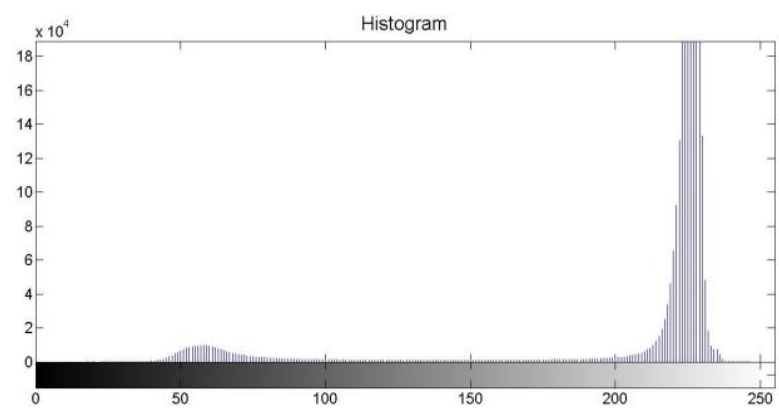

Figure 4. Histogram of rubbing image that No. 14940418

TABLE I. THRESHOLDING EVALUATION OF RUBBING IMAGES

\begin{tabular}{|c|c|c|c|c|}
\hline & Method & MSE & PSNR & $\begin{array}{c}\text { Thresholding } \\
\text { Level }\end{array}$ \\
\hline \multirow{6}{*}{$\begin{array}{c}\text { Name. } \\
\text { 12443747 } \\
\text { Size of } \\
\text { Image : } \\
2112 * 1781\end{array}$} & $\begin{array}{l}\text { Histogram } \\
\text { Isodata }\end{array}$ & 116.46 & 27.46 & 147.8 \\
\hline & $\begin{array}{l}\text { Minimum } \\
\text { cross entropy }\end{array}$ & 109.54 & 27.73 & 137.0 \\
\hline & $\begin{array}{l}\text { Kittler's } \\
\text { method }\end{array}$ & 138.76 & 26.71 & 189.0 \\
\hline & Max entropy & 97.67 & 28.23 & 121.0 \\
\hline & $\begin{array}{l}\text { Ostu's } \\
\text { method }\end{array}$ & 114.03 & 27.56 & 146.0 \\
\hline & $\begin{array}{c}\text { Fuzzy C } \\
\text { Means }\end{array}$ & 189.06 & 25.36 & 97.5 \\
\hline \multirow{6}{*}{$\begin{array}{l}\text { Name. } \\
14940418 \\
\text { Size of } \\
\text { Image : } \\
1688 * 2400\end{array}$} & $\begin{array}{l}\text { Histogram } \\
\text { Isodata }\end{array}$ & 27.41 & 33.75 & 168.18 \\
\hline & $\begin{array}{l}\text { Minimum } \\
\text { cross entropy }\end{array}$ & 18.08 & 35.56 & 133.0 \\
\hline & $\begin{array}{l}\text { Kittler's } \\
\text { method }\end{array}$ & 28.17 & 33.63 & 215.0 \\
\hline & Max entropy & 25.12 & 34.13 & 208.0 \\
\hline & $\begin{array}{l}\text { Ostu's } \\
\text { method }\end{array}$ & 19.09 & 35.32 & 149.0 \\
\hline & $\begin{array}{c}\text { Fuzzy C } \\
\text { Means }\end{array}$ & 238.9 & 24.35 & 104.5 \\
\hline
\end{tabular}

The three methods that are Histogram Isodata, Minimum cross entropy and Ostu's method have obtained similar thresholding level in No.12443747. The Max entropy method does give minimum MSE. The FCM 
method gives smaller thresholding level, and show more detail structures in segmentation rubbing image.

\section{CONCLUSIONS}

Automatic extraction of characters from Chinese rubbing images is a difficult task due to their degradation because of different types of noise. Applying a global threshold or a chosen threshold based on visual intuition might miss the finer Chinese characters with low intensity values.In this study, we make a compared evaluation of image thresholding methods for Chinese rubbing image. Several other issues remain to be addressed. For example, the increasing number of color documents becomes a new challenge for binarization and segmentation. Other topic such as that after applying global threshold, left out background image consists of some mixed image background and characters intensities on which we apply mathematical morphology (opening and closing), which produces a smooth contour and gives an adaptive threshold.

\section{ACKNOWLEDGEMENTS}

This work was supported by the National Natural Science Foundation of China (Grant No. 61472173), the grants from the Science and Technology Planning Project of Jiangxi Province of China, No. 20111BBG70032-2.

\section{REFERENCES}

[1] Roy, Bishakha, and Rohit Kamal Chatterjee. "Historical Handwritten Document Image Segmentation Using Morphology." Emerging Trends in Computing and Communication. Springer India, 2014. 123-131.

[2] Pal, Nikhil R., and Sankar K. Pal. "A review on image segmentation techniques." Pattern recognition 26.9 (1993): 1277 1294.

[3] Huang, Zhi-Kai, and Kwok-Wing Chau. "A new image thresholding method based on Gaussian mixture model." Applied Mathematics and Computation 205.2 (2008): 899-907.
[4] Sezgin, Mehmet. "Survey over image thresholding techniques and quantitative performance evaluation." Journal of Electronic imaging 13.1 (2004): 146-168.

[5] Otsu, N., "A Threshold Selection Methodfrom Gray-Level Histograms," IEEE Transactions on Systems,Man, and Cybernetics, Vol. 9, No. 1, 1979, pp. 62-66.

[6] Kittler, Josef, and John Illingworth. "Minimum error thresholding." Pattern recognition 19.1 (1986): 41-47.

[7] Ridler, T. W., and S. Calvard. "Picture thresholding using an iterative selection method." IEEE transactions on Systems, Man and Cybernetics 8.8 (1978): 630-632.

[8] Nagy, George, Sharad Seth, and Mahesh Viswanathan. "A prototype document image analysis system for technical journals." Computer 25.7 (1992): 10-22.

[9] Pham, Dzung L., and Jerry L. Prince. "An adaptive fuzzy C-means algorithm for image segmentation in the presence of intensity inhomogeneities." Pattern Recognition Letters 20.1 (1999): 57-68.

[10] Sezgin, Mehmet. "Survey over image thresholding techniques and quantitative performance evaluation." Journal of Electronic imaging 13.1 (2004): 146-168.

[11] Huang, Zhi-Kai, et al. "Using fuzzy c-means cluster for histogrambased color image segmentation." Information Technology and Computer Science, 2009. ITCS 2009. International Conference on. Vol. 1. IEEE, 2009.

[12] http://pds.lib.harvard.edu/pds/view/10401767?n=1\&imagesize=12 $00 \&$ jp2Res $=.25 \&$ print Thumbnails $=$ no

[13] http://ids.lib.harvard.edu/ids/view/14940418? width=1688\&height= 2400\&html=y

[14] Ahmed, Mohamed N., et al. "A modified fuzzy c-means algorithm for bias field estimation and segmentation of MRI data." Medical Imaging, IEEE Transactions on 21.3 (2002): 193-199.

[15] Mehri, Maroua, et al. "Texture feature evaluation for segmentation of historical document images." Proceedings of the 2nd International Workshop on Historical Document Imaging and Processing. ACM, 2013.

[16] Trier, Øivind Due, and Torfinn Taxt. "Evaluation of binarization methods for document images." IEEE Transactions on Pattern Analysis and Machine Intelligence 17.3 (1995): 312-315.

[17] Sauvola, Jaakko, and Matti Pietikäinen. "Adaptive document image binarization." Pattern recognition 33.2 (2000): 225-236. 